\title{
The sooner the better?
}

\author{
Shengshou $\mathrm{Hu}, \mathrm{MD}$, and Wei Feng, MD, $\mathrm{PhD}$
}

\author{
From the Department of Surgery, Fuwai Hospital, National Center for Cardiovascular Disease, Chinese Academy \\ of Medical Sciences and Peking Union Medical College, Beijing, China. \\ Disclosures: Authors have nothing to disclose with regard to commercial support. \\ Received for publication Nov 8, 2015; accepted for publication Nov 9, 2015; available ahead of print Dec 10 , \\ 2015. \\ Address for reprints: Shengshou Hu, MD, Department of Surgery, Fuwai Hospital, 167 Beilishi Rd, Xi Cheng \\ District, Beijing 100037, China (E-mail: shengshouhu@yahoo.com). \\ J Thorac Cardiovasc Surg 2016;151:e63-4 \\ $0022-5223 / \$ 36.00$ \\ Copyright (C) 2016 by The American Association for Thoracic Surgery \\ http://dx.doi.org/10.1016/j.jtcvs.2015.11.010
}

Ruptured mycotic aneurysms are rare among intracranial aneurysms, but they are associated with a high mortality-reaching $80 \%{ }^{1}$ It is generally accepted that in the presence of ruptured mycotic aneurysms that cause significant neurologic deterioration, urgent neurosurgical intervention is warranted. On the other hand, early cardiac surgery may be critical in high-risk patients to prevent further embolic events, including intracranial embolism. A difficult decision must be made: How soon after a neurosurgery procedure can a cardiopulmonary bypass be safely performed? In their case report, Maruyama and colleagues ${ }^{2}$ describe a 26-year-old patient who underwent a successful mitral valve repair only 3 days after a craniotomy for resection of a ruptured mycotic aneurysm.

The patient described is a young man with initial presentation of persistent high fever of unclear etiology. Further workup, including transthoracic and transesophageal echocardiography revealed moderate mitral valve regurgitation and large vegetations on both anterior and posterior mitral leaflets. Blood culture grew Haemophilus parainfluenzae. The patient also experienced extracardiac complications, including splenic infarctions, renal infarctions, and cerebral hemorrhagic infarctions in the right temporal lobe and left occipital lobe. The patient was first treated conservatively with antibiotics. However, follow-up intracranial imaging studies indicated enlargement of the hemorrhage of the left occipital lobe. An angiogram showed a ruptured left posterior cerebral artery aneurysm. The priority and timing of the craniotomy and cardiovascular surgery were carefully weighed by the neurosurgery and cardiac surgery teams. Eventually, the decision was made to first perform the craniotomy and resection of the mycotic aneurysm to minimize the risk of worsening intracranial bleeding during the cardiopulmonary bypass procedure. Three days after, mitral valve repair was performed under full heparinization. The patient was followed for 6 weeks postsurgery and showed no evidence of neurologic deficits or mitral valve regurgitation.

The treatment of infectious endocarditis has remained a challenge, particularly in patients with cardiac and

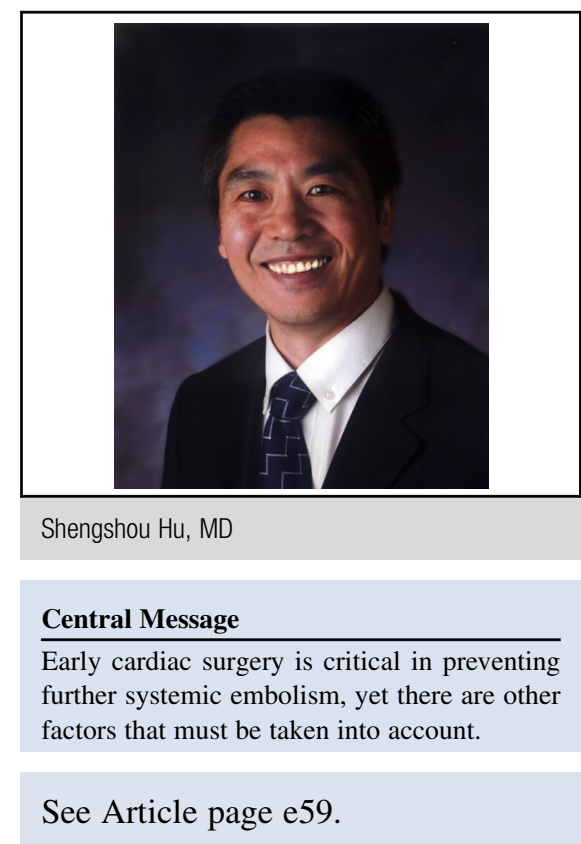

neurologic complications. In a randomized trial by Kang and colleagues, ${ }^{3}$ patients with severe valve disease and large vegetations were randomized to an early-surgery group and a conventional-treatment group. The study showed that there was significant reduction of end point death of any cause and embolic events in the earlysurgery group (ie, patients who underwent cardiac surgeries within 48 hours of randomization). However, the study excluded the patients with a history of major stroke or poor medical condition. There has been no controlled trial available to guide situations when infectious endocarditis is complicated by a ruptured mycotic aneurysm or intracranial hemorrhage. Previous literature based on retrospective reviews has suggested an interval of 4 weeks from intracranial hemorrhage to cardiac surgery. ${ }^{4}$ The recently published European Society of Cardiology guidelines of infectious endocarditis also recommend to postpone the cardiac surgery for at least 1 month in patients with intracranial hemorrhage. The major concerns of heart surgery in a patient with intracranial hemorrhage include increasing hematoma due to heparinization and compromised cerebral perfusion due to hypotension. It is therefore reasonable to consider securing intracerebral aneurysms before heart surgery.

The treatment of intracranial mycotic aneurysms can be achieved by either open craniotomy or endovascular techniques, depending on whether there is a mass effect or involvement of the eloquent areas. ${ }^{1,5}$ There has been increasing application of endovascular therapy for 
mycotic aneurysms. This procedure is less invasive and can be performed in patients who are unable to tolerate a craniotomy, or when clot evacuation is not required. ${ }^{1,5}$ Using endovascular embolization of both a ruptured and an unruptured cerebral aneurysm, Asai and colleagues ${ }^{6}$ successfully performed a heart repair 1 day later.

Early cardiac surgery is critical in preventing further systemic embolism, but there are other contributing factors that must be taken into account to improve patient outcome. An individualized and multidisciplinary approach must be adopted in clinical decision making. The case report by Maruyama and colleagues ${ }^{2}$ is a welcome addition to the literature regarding the optimal timing of surgical interventions by demonstrating that a valve repair can be safely performed only 3 days after resection of a ruptured intracranial mycotic aneurysm. Is sooner better? A prospective randomized study is required to provide a more definitive answer.
However, given the rarity of intracranial mycotic aneurysms and the variety of clinical presentations of infectious endocarditis, a controlled study may be difficult.

\section{References}

1. Ducruet AF, Hickman ZL, Zacharia BE, Narula R, Grobelny BT, Gorski J, et al. intracranial infectious aneurysms: a comprehensive review. Neurosurg Rev. 2010;33:37-46.

2. Maruyama R, Yamada A, Sugiyama T, Ujihira K, Nishioka N, Iba Y, et al. Mitral valve repair for endocarditis can be performed 3 days after repair of a bleeding mycotic brain aneurysm. J Thorac Cardiovasc Surg. 2016;151:e59-61.

3. Kang DH, Kim YJ, Kim SH, Sun BJ, Kim DH, Yun SC, et al. Early surgery versus conventional treatment for infective endocarditis. N Engl J Med. 2012;366: 2466-73.

4. Rossi M, Gallo A, De Silva RJ, Sayeed R. What is the optimal timing for surgery in infective endocarditis with cerebrovascular complications? Interact Cardiovasc Thorac Surg. 2012;14:72-80.

5. Kannoth S, Thomas SV. Intracranial microbial aneurysm (infectious aneurysm): current options for diagnosis and management. Neurocrit Care. 2009;11:120-9.

6. Asai T, Usui A, Miyachi S, Ueda Y. Endovascular treatment for intracranial mycotic aneurysms prior to cardiac surgery. Eur J Cardiothorac Surg. 2002;21:948-50.

\title{
EDITORIAL COMMENTARY
}

\section{Multidisciplinary risk assessment and treatment: The evolution of triage}

\author{
William E. Stansfield, MD
}

\footnotetext{
From the Department of Surgery, Toronto General Hospital, Toronto, Ontario, Canada. Disclosures: Author has nothing to disclose with regard to commercial support.

Received for publication Nov 22, 2015; accepted for publication Nov 25, 2015; available ahead of print Jan 13, 2016.

Address for reprints: William E. Stansfield, MD, Department of Surgery, Toronto General Hospital, 200 Elizabeth

St, 4N-476, Toronto, ON, Canada M5G 2C4 (E-mail: william.stansfield@uhn.ca).

J Thorac Cardiovasc Surg 2016;151:e64-5

$0022-5223 / \$ 36.00$

Copyright (c) 2016 by The American Association for Thoracic Surgery

http://dx.doi.org/10.1016/j.jtcvs.2015.11.053
}

Napoleon's chief surgeon, Baron Dominique Jean Larrey, is widely credited with the origin of triage. ${ }^{1}$ In the early 1800 s, Baron Larrey convinced military leadership of the need to evacuate wounded from the battlefield while conflict was still underway. This novel approach marked a major turning point in the evolution of surgical care. After all, no commander would have risked additional personnel evacuating the wounded were the care provided not actually beneficial! These benefits that we take for granted today were clearly less apparent in the early 19th century. Another novel approach was to treat the most critically injured first, without regard for nationality or rank. This undoubtedly helped establish medical prioritization as a vital triage function.

Writing in 1846 , in what must have been a colder, more rational time, British naval surgeon John Wilson further

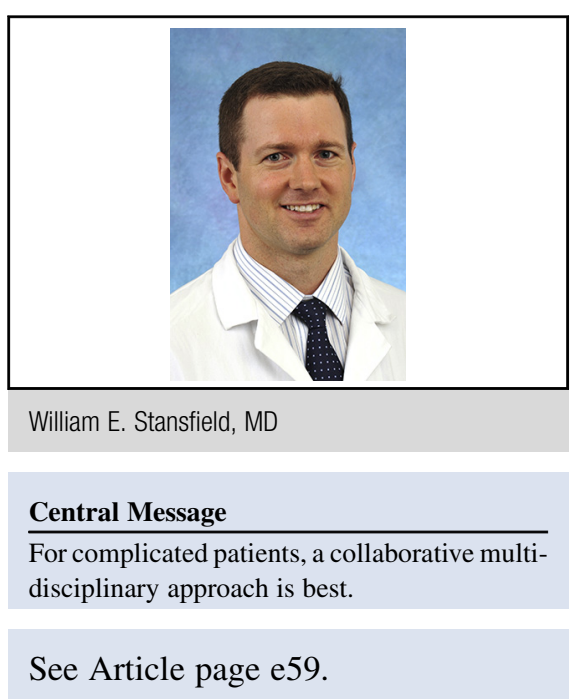

refined the concepts of triage and prioritization. ${ }^{2}$ He limited evacuations to those who might benefit from surgical care. This model distributed surgical decision making to the corpsmen, who were closer to the front line. Importantly, it implies a broader knowledge and understanding of surgical capabilities among the nonsurgeon members of the team. 